\title{
Cervical Motion Preservation Surgery: Why Sometimes Failed? A Biomechanical Point of View
}

\section{Alessandro Landi*}

Department of Neurology and Psychiatry, Division of Neurosurgery, University of Rome Sapienza, Italy

Anterior cervical discectomy and fusion (ACDF) with cage is actually an established surgical procedure for treating cervical disc disease. Good clinical outcome, radiological results and long term follow up demonstrated its efficacy [1,2]. Notwithstanding, the risk of an adjacent disc disease (ASD) and the need to preserve the physiological metameric movement has led to the development of new devices able to substitute cervical arthrodesis through an anterior approach; the aim of these devices is to restore the range of motion of the involved vertebral segment, thus the technique most commonly used is the total disc replacement.

Despite the excellent clinical results obtained in the short term follow-up which confirmed the high range of motion given by the prosthesis implanted in the involved vertebral space [1,3-5], long-term follow-up has raised some doubts about the use of these systems [4,5]. In fact, some of these devices have needed revision surgery after several years $[6,7]$. The causes of failure seem to be: progression of neurological symptoms, infections, heterotopic ossification or mobilization of the prosthesis. So why does it happen? Why is there a progression of symptoms? How come the prosthesis ossify?

In the 2012 a literature review was done about the results of the total disk replacement in terms of wear and durability; this review showed that the materials of the prosthesis and biomechanical tests in vitro give very good results in terms of motility predictable for 50 years, but no study was performed in terms of which grade of involvement the artificial motion gives to the remaining structures of the vertebral segment [8]. Kim et al. [9] hypothesized that the prosthesis can influence the biomechanical sagittal alignment of the cervical spine. Other studies have shown that there is a higher rate of heterotopic ossification in multilevel systems than in single-level, hypothesizing that this is a result of a high grade degeneration of the metameres involved [10].

In my opinion, technological innovations have led over time to the development of various kinds and different materials of discal prosthesis, but the main goal of the devices has always been to preserve or restore the range of motion of the segment.

One aspect often overlooked, however, is that the disc is an articulation with the biomechanical properties of shock absorber; in fact, the fibrous lamellar structure of the annulus with high elastic module, give elastic resistance to excessive degrees of movement especially in rotation. In particular we have to highlight that articular masses are the most stressed and involved joints in the movements of flexion-extension and axial rotation, which are anatomically responsible for these movements. The discal prosthesis are constrained to a range of motion greater than that of the normal disc, in relation to the fact that the prosthesis have not the elastic resistance exerted by the fibers of the annulus especially in the movement of axial rotation. This implies a functional overload of the facet joints which undergo progressive degeneration. In addition, when the prosthesis is inserted, the LLA has to be removed. LLA is an essential structure for the cervical spine stability, especially for the flexion and extension movement. The loss of the LLA elastic resistance in the extension movements leads to a further functional overload of the articular masses, which are already stressed by the prosthesis during axial rotation. Since we are talking about spondylodiscoarthrosis, that is the clinical and radiological manifestation of the unstable phase of the degenerative cascade, we are facing a treatment that does nothing but increase the segmental instability, causing and not preventing the evolution of the degenerative cascade. In addition, it was found that the incidence of adjacent segment disease in long-term follow up is similar in patients treated with prosthesis compared to those treated with anterior fusion [1]. This disproves the fact that the total disc replacement prevents the ASD. In this regard I think that the possibility of failure of prosthetic surgery is closely related to cervical pathology. It is therefore important the selection of the patient for the choice of treatment with cervical disc prosthesis, which have to be young, with preserved physiological lordosis, affected by a soft hernia without radiological signs of instability and a single level disease. In these cases, the preservation of motion obtained good results in my opinion. As reported in the literature $[10,11]$, it is important to exclude the multilevel pathology that is sign of an advanced stage of spondylo discarthrosis, no longer susceptible of prosthetic surgery. Moreover, the choice of materials is important, because it should mimic as much as possible the characteristics of elastic resistance to the movement of the disc.

In my opinion these are the main features to have a good prognostic outlook in cervical prosthetic surgery. Actually, however, there are not devices that completely mimic the elastic resistance of the disc with controls of long-term follow up.

Currently none of the devices are able to restore $100 \%$ functional units responsible of the movement of the motor unit. In fact, since the segment of motion involves the disk, the joint structures, the ligaments and the posterior muscles, the prosthetic have to include all the structures responsible for movement. This because the disc and LLA and LLP ligaments are responsible for the passive resistance to excessive degrees of movement, while the joint structures are responsible for active movements of the vertebral body.

Clearly, surgery with preservation of the motion is the future of spinal stabilization, but it has to be understood not as a preservation surgery, but as a neutralization of excessive degrees of motion surgery, which is one of the causes of the metameric degenerative cascade.

\section{References}

1. Mummaneni PV, Amin BY, Wu JC, Brodt ED, Dettori JR, et al. (2012) Cervical artificial disc replacement versus fusion in the cervical spine: a systematic

*Corresponding author: Dr. Alessandro Landi, MD, PhD, Department of Neurology and Psychiatry, Division of Neurosurgery, Viale Del Policlinico 15500181 , University of Rome Sapienza, Rome, Italy, Tel: +39 329 0641772; Fax: +39 06 4997911; E-mail: dott.alessandro.landi@gmail.com

Received January 31, 2013; Accepted February 01, 2013; Published February 04, 2013

Citation: Landi A (2013) Cervical Motion Preservation Surgery: Why Sometimes Failed? A Biomechanical Point of View. J Spine S3: e001. doi:10.4172/2165-7939. S3-e001

Copyright: (c) 2013 Landi A. This is an open-access article distributed under the terms of the Creative Commons Attribution License, which permits unrestricted use, distribution, and reproduction in any medium, provided the original author and source are credited. 
Citation: Landi A (2013) Cervical Motion Preservation Surgery: Why Sometimes Failed? A Biomechanical Point of View. J Spine S3: e001. doi:10.4172/2165-7939.S3-e001

review comparing long-term follow-up results from two FDA trials. Evid Based Spine Care J 3: 59-66.

2. Marotta N, Landi A, Tarantino R, Mancarella C, Ruggeri A, et al. (2011) Five-year outcome of stand-alone fusion using carbon cages in cervical disc arthrosis. Eur Spine J 20: S8-12.

3. Boselie TF, Willems PC, van Mameren H, de Bie R, Benzel EC, et al. (2012) Arthroplasty versus fusion in single-level cervical degenerative disc disease. Cochrane Database Syst Rev 9: CD009173.

4. Lee JH, Jung TG, Kim HS, Jang JS, Lee SH (2010) Analysis of the incidence and clinical effect of the heterotopic ossification in a single-level cervical artificial disc replacement. Spine J 10: 676-682.

5. Pickett GE, Sekhon LH, Sears WR, Duggal N (2006) Complications with cervical arthroplasty. J Neurosurg Spine 4: 98-105

6. Jin YJ, Park SB, Kim MJ, Kim KJ, Kim HJ (2013) An analysis of heterotopic ossification in cervical disc arthroplasty: a novel morphologic classification of an ossified mass. Spine J.
7. Brenke C, Scharf J, Schmieder K, Barth M (2012) High prevalence of heterotopic ossification after cervical disc arthroplasty: outcome and intraoperative findings following explantation of 22 cervical disc prostheses. J Neurosurg Spine 17 141-146.

8. Lehman R, Bevevino AJ, Brewer DD, Skelly AC, Anderson PA (2012) A systematic review of cervical artificial disc replacement wear characteristics and durability. Evid Based Spine Care J 3: 31-38.

9. Kim SW, Shin JH, Arbatin JJ, Park MS, Chung YK, et al. (2008) Effects of a cervical disc prosthesis on maintaining sagittal alignment of the functiona spinal unit and overall sagittal balance of the cervical spine. Eur Spine J 17: 20-29.

10. Wu JC, Huang WC, Tsai TY, Fay LY, Ko CC, et al. (2012) Multilevel arthroplasty for cervical spondylosis: more heterotopic ossification at 3 years of follow-up. Spine (Phila Pa 1976) 37: E1251-1259.

11. Wu JC, Huang WC, Tsai HW, Ko CC, Fay LY, et al. (2012) Differences between 1 - and 2-level cervical arthroplasty: more heterotopic ossification in 2-level disc replacement: Clinical article. J Neurosurg Spine 16: 594-600.
This article was originally published in a special issue, Advanced Techniques in Spine Surgery handled by Editor. Dr. Alessandro Landi, University of Rome Sapienza, Italy 